\title{
A decision support system for stress only myocardial perfusion scintigraphy may save unnecessary rest studies
}

\author{
Tagil $K^{1,2}$, Jakobsson $D^{2}$, Lomsky $M^{3}$, Marving J ${ }^{4}$, Svensson $S-E^{5}$, Wollmer $P^{1}$, Hesse $B^{6}$ \\ 1. Department of Clinical Sciences, Clinical Physiology and Nuclear Medicine Unit, Malmö University Hospital, Sweden. 2. \\ EXINI Diagnostics, Ideon Science Park, Lund, Sweden. 3. Department of Clinical Physiology, Sahlgrenska University \\ Hospital, Gothenburg, Sweden. 4. Department of Clinical Physiology, Gentofta Hospital, Copenhagen, Denmark. 5. \\ Department of Clinical Physiology, Karlskrona Hospital, Sweden. 6. Clinic of Clinical Physiology and Nuclear Medicine and \\ PET, Rigshospitalet, University of Copenhagen, Denmark
}

Correspondence: Tagil K. Address: Department of Clinical Sciences, Clinical Physiology and Nuclear Medicine Unit, Malmö University Hospital, Sweden. Email: kristina.tagil@gmail.com

Received: J une 30, 2012

DOI : $10.5430 /$ jbgc.v3n2p46
Accepted: August 31, 2012

Online Published: February 22, 2013

\section{Abstract}

Aim: It is often a practical question whether to continue with the rest study after termination of a fairly normal stress myocardial perfusion scintigraphy (MPS), in particular for physicians with limited experience. The purpose of this study was to analyze the value of using a decision support system (DSS) to guide less experienced physicians in this situation.

Methods: Nine residents from eight different nuclear medicine departments interpreted 100 MPS stress studies, first without and then with access to the advice of a DSS. Each study was interpreted regarding the necessity of adding a rest study for correct interpretation of the MPS. The patients had undergone a gated stress and rest MPS, using a Tc-99m sestamibi protocol. Interpretations made by three nuclear cardiology experts, having access to all available clinical and image information, were used as the gold standard.

Results: In the cases where the gold standard interpretation wanted a rest study the 9 residents asked for it in $94 \%$ and $95 \%$ before and after having access to the DSS, respectively ( $p>0.05$ ). The residents did not want a rest study in $57 \%$ (without) and $69 \%$ (with the advice from the decision support system), in the patients, considered to have a normal stress study by the experts $(p<0.005)$. The DSS significantly reduced interobserver variation among the residents.

Conclusion: The present study shows that with the support of a DSS less experienced physicians get closer to the decisions of highly experienced nuclear cardiologists regarding the need of adding a rest study to a stress MPS.

\section{Key words}

Coronary artery disease, Decision support system, Myocardial perfusion scintigraphy, Neural networks

\section{Introduction}

If a stress study in myocardial perfusion scintigraphy (MPS) is interpreted as normal, it means that the distribution of myocardial perfusion reserve is considered to be normal. The rest study is then unnecessary and can be safely omitted. The advantages of performing only a stress study include a faster throughput of patients, better cost-efficiency, and a reduction 
of radiation exposure both for the patient and for the staff. In some patients the risk of overlooking a perfusion defect is considered too high if the stress study is not "convincingly normal", i.e. the interpreting physician cannot exclude that at rest the perfusion distribution may become more even indicating the presence of a reversible perfusion defect. When deciding whether to rely only on the stress images, in particular two parameters have to be taken into consideration: "normal" variations and the risk of attenuation artifacts, which both may give rise to a false positive regional perfusion defect. The normal variations are reflected by the variations in reference populations of activity distribution in MPS reference data ${ }^{[1]}$. Attenuation artifacts may often be discriminated from true defects by attenuation correction, with coronary angiography as gold standard ${ }^{[2]}$. Heller et al ${ }^{[3]}$ found that the use of attenuation correction increased the number of definitely normal stress studies from $20 \%$ to $50 \%$ in a group of 90 patients interpreted by ten expert nuclear cardiologists using attenuation correction.

The decision to omit the rest study after a near-normal stress study varies with the experience and with the more or less "courageous" mind of the reader. It also varies with the image quality and software tools available for interpretation of possible artifacts and with biological variations. In the present investigation residents in nuclear medicine should decide about the necessity of adding a rest study after interpretation of a stress study, first without and then with a decision support system (DSS), developed for the specific purpose of relying only on a stress study. The aim of this investigation was to determine whether such a DSS could improve the residents' decisions, the gold standard being the consensus of three nuclear cardiology experts' readings.

\section{Material and methods}

\subsection{Patients}

One hundred consecutive MPS studies from patients were selected according to criteria described below from 1200 consecutive patients undergoing MPS at Sahlgrenska University Hospital between September 15th 2005 and September 14th 2006. The study group included 53 men and 47 women, mean age 62 years, range 31-84. Demographic data are shown in table 1. The patients fulfilled the following two inclusion criteria: the MPS study should be technically successful and complete stress and rest images should be available to our three experts for interpretation, providing the gold standard. The exclusion criteria included: scintigraphic signs of LV dilatation or RV hypertrophy and obvious artifacts or technical problems in the images. The data sets of those 100 cases were distributed for interpretation among nine residents from eight different nuclear medicine departments in Sweden or Denmark. None of the patients in the study group had been included in the dataset used for training the DSS. The study was approved by the Research Ethics Committee at Gothenburg University.

Table 1. Clinical details data of the 100 patients selected

\begin{tabular}{ll}
\hline $\mathbf{1 0 0}$ patients with low/intermediate risk of CAD studies by stress / rest MPS & Numbers \\
\hline Men / women & $53 / 47$ \\
Referred for diagnosis / management of CAD & $72 / 28$ \\
Smokers / non-smokers & $15 / 85$ \\
Diabetes / hypertension / hypercholesterolaemia & $10 / 53 / 53$ \\
History of IHD (including PCI and CABG) / no history of IHD & 68 (20 and 14)/32 \\
\hline
\end{tabular}

Note. Coronary artery disease (CAD), myocardial perfusion scintigraphy (MPS), percutaneous coronary intervention (PCI), coronary Artery Bypass Graft Surgery (CABG), ischemic heart disease (IHD)

\subsection{Myocardial perfusion scintigraphy}

The patients were stressed using either maximal exercise as a symptom-limited ergometry test (56\%) or a vasodilator (adenosine) test (44\%) with the patient lying supine during adenosine infusion over six minutes. The exercise or pharmacological stress was continued for at least two minutes after injection of the tracer. The stress and rest studies were 
performed as a two-day protocol using 99mTc-sestamibi. Image acquisition began approximately 60 minutes after injection of $600 \mathrm{MBq} 99 \mathrm{mTc}$-sestamibi. Images were acquired with two different dual-head SPECT cameras (Infinia or Hawkeye, General Electric Medical Systems, Milwaukee, WI, USA) equipped with low energy, high resolution collimators. The planar projection images were acquired in the step and shoot mode using a circular rotation with the patient in a supine position. Acquisition was performed in a 64×64 matrix applying either 64 projections over 180 degrees for 40 seconds per projection or 68 projections over 204 degrees for 40 seconds per projection. In patients weighing more than $90 \mathrm{~kg}$, the acquisition time per projection was increased to 55 seconds. A zoom factor of 1.28 was used. Tomographic reconstruction was performed using filtered back-projection (Butterworth filter with critical frequency $0.52 \mathrm{cycles} / \mathrm{cm}$ and order 5 in non-gated images, and with critical frequency $0.40 \mathrm{cycles} / \mathrm{cm}$ and order 10 in gated images). An automatic motion correction program was applied where patient motion during acquisition was apparent ${ }^{[4]}$. No attenuation/scatter correction was applied.

\subsection{I mage interpretation}

Image interpretation without DSS: In the first part of the study the participating physicians received a CD (CD1) with the MPS images of only a stress study in the 100 patients. Each physician interpreted the MPS stress study and decided whether a rest study should also be performed for correct interpretation, in order to report whether the abnormality in activity distribution was assumed to be normal or to show a possible myocardial perfusion defect. If a stress perfusion defect is believed to be present, a rest study is needed to determine whether the defect is reversible or irreversible. If the stress image is believed to be normal or if an activity defect is believed not to represent a true perfusion defect, the rest test should be omitted. A software package with the EXINI heart program for display of the MPS data showing the stress short axis (SA), vertical long axis (VLA) and horizontal long axis (HLA) slices as well as the polar plot was used. The result of the physician's decision was stored in an excel file and sent back to the main investigator after this first part of the study.

Image interpretation with DSS: After 3 months each physician received another CD (CD2) with the stress studies of the same 100 patients and the EXINI software program described above. The CD now also included a tool giving an advice regarding interpretation of stress studies only, i.e. recommending whether the stress study could be considered normal so that it was safe to skip the rest study, or abnormal so that a rest study should be performed. Each physician again interpreted the MPS stress studies and made his or her decision about the necessity of having also a rest study performed but the decision now also based on the advice from the DSS in the EXINI heart program. The data available on the computers were the stress SA, VLA, HLA slices as well as the polar plots on both occasions but with no information on clinical data except gender. These second interpretations from the physicians were stored in another excel file and sent back to the main investigator.

\subsection{The EXI NI heart program}

The EXINI heart software package (version 4.2.1; EXINI Diagnostics AB, Lund, Sweden) was used in this study. The DSS used for automated interpretation of the "MPS stress only images" was based on automated image processing algorithms, ANN and a database of classified MPS images from the Royal Brompton Hospital in London ${ }^{[5,6]}$.

When the program was developed and validated its capability not to omit a rest study when indicated was $99 \%$ and its ability not to perform a rest study when not indicated $53 \%$.

\subsection{Gold standard}

The interpretations by three senior nuclear cardiologists with more than 25 years of experience were used as the gold standard regarding presence or absence of a stress perfusion defect (which might represent ischaemia and/or infarction). The software package used by the experts was also the display function in the EXINI heart program, including the slices of the stress and rest SA, VLA and HLA axes as well as the polar plots. No quantification data, based on reference databases, were available. The only quantified data shown were the count values in percent of the regional maximum value of the left 
ventricular walls. The experts' conclusions were based only on the evaluation of the images, in 90 cases total consensus was obtained regarding the absence or presence of a regional perfusion defect during stress. In the remaining 10 cases a majority decision (2/3) was accepted.

\subsection{Test physicians}

Nine residents in nuclear medicine from Denmark ${ }^{[3]}$ and Sweden ${ }^{[6]}$ participated in the study (testing physicians group). The test physicians were residents with experience of MPS interpretation between six months and four years of training.

\subsection{Statistical methods}

The statistical analysis was made using generalized linear mixed models. Here both physicians and patients are treated as random effects. The effect of a DSS was estimated using logistic mixed regression (two random effects for each physician and one for each examination). Analysis was performed with the software R package lme4 ver. 0.999375-32. $P$-values less than 0.05 were considered significant.

\section{Results}

Of the 100 MPS studies the consensus of our three expert nuclear cardiologists found that 57 MPS stress images were normal (no regional perfusion defects) and 43 were abnormal, with a defect that could represent infarction or stressinduced ischemia (see Table 2). In the group regarded as abnormal 23 patients had been stressed with exercise and 20 patients with adenosine.

The nine residents suggested a rest study should be performed in 65 patients (mean value, range 54-84) without the advice of DSS. After the advice from the DSS the residents reduced their recommendation to 59 patients (mean value, range 48-66), i.e. a $9 \%$ decrease $(p=0.03)$.

In the subgroup of 57 patients regarded by the experts as having normal stress-rest MPS, the residents suggested to add a rest study significantly less, although with considerable variations (from 24 (14-42) to 18 (8-23)) after the advice from the DSS. Two of the residents actually increased their use of rest studies among these "normal cases" (see Figure 1A). The decision not to ask for a rest study in patients increased from $61 \%$ to $72 \%$, an increase of $18 \%(p=0.03)$ leading to a decrease in interobserver variability.

Asking for a rest study, when indicated, was decided correctly by the nine physicians in $94 \%$ of the cases in their first interpretation and 95\% in their second DSS assisted interpretation and only showing minor variations (see Figure 1B).

Table 2. The different requirements of the physicians for a rest study according to their interpretations of the stress MPS as being normal or abnormal

\begin{tabular}{|c|c|c|c|c|c|c|}
\hline \multirow{2}{*}{$\begin{array}{l}\text { Number of patients } \\
\text { assessed }\end{array}$} & \multirow{2}{*}{ Gold standard } & \multirow{2}{*}{ EXINI with DSS } & \multicolumn{2}{|c|}{$\begin{array}{l}\text { Residents' interpretations } \\
\text { (mean values and ranges) }\end{array}$} & \multirow{2}{*}{ Change* (\%) } & \multirow{2}{*}{$p^{*}$} \\
\hline & & & $1^{\text {st }}$ (without DSS) & $2^{\text {nd }}$ (with DSS) & & \\
\hline Rest study not needed & 57 & 45 & $\begin{array}{l}35 \\
\text { (range16-46) }\end{array}$ & $\begin{array}{l}41 \\
\text { (range 34-52) }\end{array}$ & Increase 18 & 0.03 \\
\hline Rest study needed & 43 & 55 & $\begin{array}{l}65 \\
\text { (range 54-84) }\end{array}$ & $\begin{array}{l}59 \\
\text { (range 48-66) }\end{array}$ & Decrease 9 & 0.03 \\
\hline
\end{tabular}

Note. Gold standard: Consensus evaluation by the three experts. EXINI with DSS: recommendation by the software whether necessary to proceed with a rest study due to the stress study interpreted by the DSS as not clearly normal. * refers to changes between first and second interpretation. Myocardial perfusion scintigraphy (MPS), decision support system (DSS). 


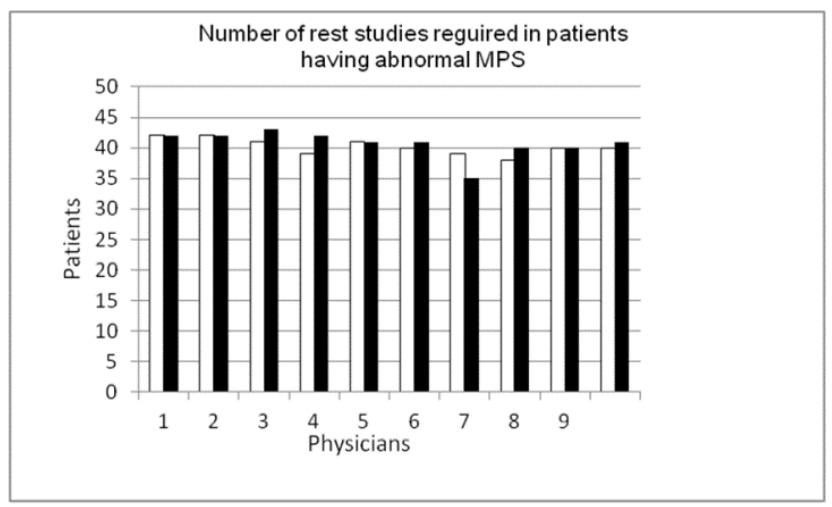

A

Figure 1. A: Patients assessed to show normal stress myocardial perfusion scintigraphy by expert consensus. B: Patients assessed to show abnormal stress myocardial perfusion scintigraphy by expert consensus.

White bars: The first nine bars are the number of rest studies suggested to be performed by the nine less experienced physicians without DSS advice. The tenth white bar is the number of rest studies suggested to be performed of an average of the 9 physicians. Black bars: Number of rest studies suggested to be performed by the nine, less experienced physicians with DSS advice. The tenth black bar is the number of rest studies suggested to be performed of an average of the 9 physicians.

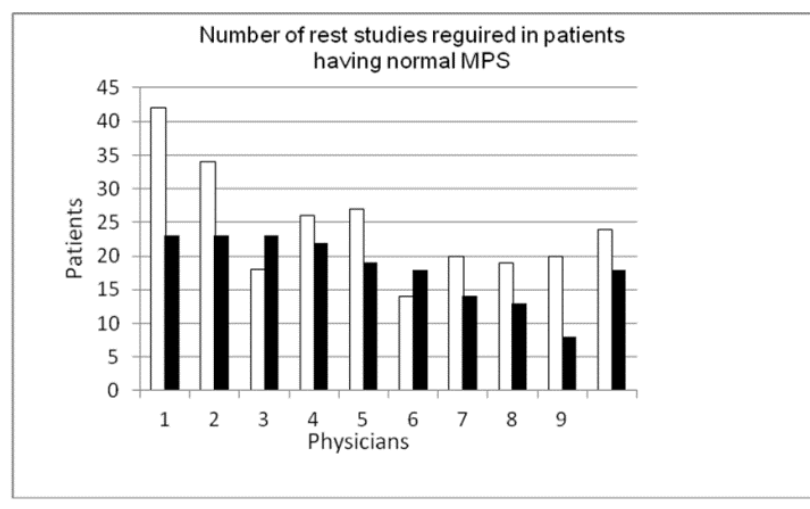

B

\section{Discussion}

A stress only MPS is currently considered the state-of-art protocol in patients undergoing MPS if the stress images are normal. Addition of an unnecessary rest study increases the radiation exposure to the patient and the staff by $100 \%$ (or more in one-day protocols), and time and money are wasted which enhances the aim of using stress-only protocol ${ }^{[7]}$. Our study shows that an advice from a DSS in a population comprising patients with low to intermediate likelihood of CAD can improve the correct choice of a stress-only protocol for less experienced nuclear medicine physicians. The improvement is important because less trained physicians such as residents often have to make this choice in the clinical routine. Furthermore the support of the system reduced the inter-observer variation among the less experienced physicians for this patient group.

The improvement in reducing unnecessary rest studies was not undermined by losing necessary rest studies. The physicians had, already without an advice from the DSS, a fairly low threshold for requiring a rest study in patients considered by the experts to have an abnormal stress MPS. This "safe strategy" was unchanged, probably because the DSS also gave recommendations in a rather safe direction in these patients.

The choice of gold standard for MPS has been debated. The CAG used to be the obvious gold standard for the diagnosis of coronary artery disease including MPS interpretation, but it has repeatedly been shown that MPS is more closely related than CAG to prognosis in stable coronary disease ${ }^{[8]}$. This strongly suggests that CAG is inferior to MPS regarding true information in some respects of coronary disease. Human expert evaluation has become popular, and in studies like the 
present one it also appears to be a highly relevant gold standard, since the aim of the decision support is to bring the interpretation up to the optimal, i.e. the expert level. This is also in agreement with guidelines' recommendations that skipping the rest study must be based on a decision taken by an experienced physician ${ }^{[9,10]}$. However, as mentioned above this is quite often not possible to follow in practical, clinical life. The design of our study was chosen to mimic a routine workflow and suggest a practical solution to this suboptimal, but realistic routine.

The DSS has to be seen as an additional tool in the situation whether to continue with the rest study or not. The present investigation was performed in a group of consecutive patients with a low to intermediate likelihood of CAD, in whom there is a fair chance to save unnecessary rest studies. Other factors may also be helpful in the discrimination between normal and abnormal MPS studies in such patients including attenuation correction, gating, image acquisition in the prone position and increased activity injected.

Attenuation correction: A low cardiac event rate was found among patients with normal stress MPS in a study regarding stress-only studies performed with attenuation correction ${ }^{[1]}$. Studies also showed that attenuation correction was more effective in correcting false inferior wall defects compared to anterior defects and was more important in men compared to women ${ }^{[12]}$. Despite the fact that a number of studies show positive results when using attenuation correction, other issues such as standardization and cost-effectiveness must also be considered ${ }^{[13]}$. Furthermore new artifacts can be created from the attenuation correction process ${ }^{[14]}$ which may be troublesome and not entirely harmless especially for the inexperienced interpreter. An increase in image acquisition time in obese patients may have limited the complicated interpretation related to attenuation artifacts in overweight patients. However, a sub-analysis did not disclose any relationship between body weight and "unnecessary" rest studies.

Prone position: The use of adding prone images may help in case of attenuation artifacts ${ }^{[12]}$, though it should not be used as the only projection and therefore considerably increases acquisition time ${ }^{[15]}$, often breaking logistics. Thus it is rather demanding both for the staff and for the patients.

Gating of the stress study giving the possibility of wall motion and wall thickening evaluation ${ }^{[16]}$ is another parameter that may be of help in the discrimination between perfusion defects and attenuation artifacts. Though of value in the final interpretation, wall motion and wall thickening does not exclude a stress perfusion defect since the perfusion image represents the myocardium during stress with or without a stress-induced perfusion defect, but wall motion and wall thickening may have recovered in the time interval between tracer injection and imaging.

Activity injected: Finally an increased amount of activity injected might be helpful in noisy images. Some studies suggest that a higher technetium activity injected for the stress study ${ }^{[17]}$ is to prefer if the stress-only strategy is introduced to the clinic while others found lower doses sufficient ${ }^{[18]}$. The current trend is rather to use even lower activities than those used in the present study in order to reduce radiation exposure.

In addition to possible technical options during acquisition, access to a DSS tool can facilitate the correct decisions in the difficult cases, in particular of value for less experienced physicians. The EXNI software used in this study has the acquired feature designed to provide stress-only recommendations. The software uses a database of images required from patients interpreted by experts as having normal MPS. Algorithms were trained using artificial neural networks and a database containing both normal and abnormal MPS studies regarding ischaemia and infarction. The system was created in the same way as a previous one dealing with both stress and rest images ${ }^{[19]}$. As far as we know no other commercially available MPS interpretation programs offer this specific function.

Limitations: Availability of attenuation corrections might have reduced the influence of the DSS to some degree, both for bringing the residents' interpretation closer to that of the experts and possibly also to each others interpretation. On the other hand the absence of attenuation correction reflects the situation in 2000-2005, when only $5 \%$ of the entire base of 
SPECT cameras were equipped with attenuation correction ${ }^{[20]}$. Now in 2011 the number has increased, but still a large number of the SPECT cameras in use for MPS do not include the option for attenuation correction.

The lack of clinical information for the residents would most likely not have made any difference, since the decision of going on with a rest study has to rely on the question of a possible demonstration of a regional perfusion defect during stress, and this can hardly be rejected in a patient referred to a MPS indicated on clinical suspicion of coronary disease. Finally the skills of the residents might have improved slightly due to further training in the interval between the first and second readings. However, three months interval during summer including holidays will hardly have a big impact.

\section{Conclusions}

Our study shows that an advice from a DSS significantly improves the decision of less trained nuclear medicine physicians regarding the demand for a rest study after a normal or near-normal stress MPS in patients with low to intermediate likelihood of CAD. The "safety first attitude of the residents - better have a rest study if in doubt" - was unaffected in the group of patients, considered indicated by the experts. The inter-observer variation among the residents significantly decreased and got closer to the consensus decisions of the three expert interpretations.

\section{Conflict of interest}

Kristina Tägil and David Jakobsson are shareholders in EXINI Diagnostics AB, which owns a neural network based decision support system for analysis of myocardial perfusion images.

\section{Acknowledgement}

The authors would like to recognize the scientific contributions, as well as friendship, of Dr Jens Marving who died during the preparation of this manuscript. The authors would also like to thank Ulrik Hesse, who guided and assisted us with the statistical part of the study.

\section{References}

[1] Svensson A, Akesson L, Edenbrandt L. Quantification of myocardial perfusion defects using three different software packages. Eur J Nucl Med Mol Imaging. 2004 Feb; 31(2): 229-32. PMid:15129705 http://dx.doi.org/10.1007/s00259-003-1361-4

[2] Kjaer A, Cortsen A, Rahbek B et al. Attenuation and scatter correction in myocardial SPET: improved diagnostic accuracy in patients with suspected coronary artery disease. Eur J Nucl Med Mol Imaging. 2002 Nov; 29(11): 1438-1442. PMid:12397461 http://dx.doi.org/10.1007/s00259-002-0932-0

[3] Heller GV, Bateman TM, Johnson LL et al. Clinical value of attenuation correction in stress-only Tc-99m sestamibi SPECT imaging. J Nucl Cardiol. 2004; 11: 273-81. PMid:15173774 http://dx.doi.org/10.1016/j.nuclcard.2004.03.005

[4] Matsumoto N, Berman DS et al. Quantitative assessment of motion artifacts and validation of a new motion-correction program for myocardial perfusion SPECT. J Nucl Med. 2001; 42: 687-94. PMid:11337561

[5] Gjertsson P, Lomsky M, Richter J, Ohlsson M, Tout D, van Aswegen A, et al. The added value of ECG-gating for the diagnosis of myocardial infarction using myocardial perfusion scintigraphy and artificial neural networks. Clin Physiol Funct Imaging. 2006; 26: 301. PMid:16939508 http://dx.doi.org/10.1111/j.1475-097X.2006.00694.x

[6] Lomsky M, Richter J, Johansson L, Hoilund-Carlsen PF, Edenbrandt L. Validation of a new automated method for analysis of gated-SPECT images. Clin Physiol Funct Imaging. 2006; 26: 139. PMid:16640507 http://dx.doi.org/10.1111/j.1475-097X.2006.00667.x

[7] Bhavnani SP, Heller GV. Stress-only myocardial perfusion imaging...it IS time for a change! J Nucl Cardiol. 2011 ; 18: 836-9. PMid:21818699 http://dx.doi.org/10.1007/s12350-011-9432-6

[8] Shaw LJ, Iskandrian AE. Prognostic value of gated myocardial perfusion SPECT. J Nucl Cardiol. 2004: 11: $171-185$. PMid:15052249 http://dx.doi.org/10.1016/j.nuclcard.2003.12.004 
[9] Des Prez RD, Dahlberg ST, Einstein AJ et al. Stress only myocardial perfusion imaging. ASNC Announcement. J Nucl Cardiol 2009:16:329. Henzlova MJ, Cerqueira MD, Mahmarian JJ et al. ASNC imaging guidelines for nuclear cardiology procedures: Stress protocols and tracers. J Nucl Cardiol. 2006; 13: e80-90.

[10] Henzlova MJ, Cerqueira MD, Mahmarian JJ et al. ASNC imaging guidelines for nuclear cardiology procedures: Stress protocols and tracers. J Nucl Cardiol. 2006; 13: e80-90. PMid:17174798 http://dx.doi.org/10.1016/j.nuclcard.2006.08.011

[11] Gibson PB, Demus D, Noto R et al. Low event rate for stress-only perfusion imaging in patients evaluated for chest pain. J Am Coll Cardiol. 2002; 39: 999-1004. http://dx.doi.org/10.1016/S0735-1097(02)01720-5

[12] Malkerneker D, Brenner R, Martin WH et al. CT-based attenuation correction versus prone imaging to decrease equivical interpretations of rest/stress Tc-99mtrtrofosmin SPECT MPI. J Nucl Cardiol. 2007; 14: 314-23. PMid:17556165 http://dx.doi.org/10.1016/j.nuclcard.2007.02.005

[13] Germano G, Slomka P J, Berman D S. Attenuation correction in cardiac SPECT: The boy who cried wolf? J Nucl Cardiol. 2007; 14: 25-35. PMid:17276303 http://dx.doi.org/10.1016/j.nuclcard.2006.12.317

[14] Cuocolo A. Attenuation correction for myocardial perfusion SPECT imaging: still a controversial issue. Eur J Nucl Med Mol Imaging. 2011; 38: 1887-1889. PMid:21874324 http://dx.doi.org/10.1007/s00259-011-1898-6

[15] Germano G, Slomka PJ, Berman DS. Supine acceptance of a conventional imaging position may make you less prone to success. J Nucl Cardiol. 2010; 17: 16-18. PMid:20035389 http://dx.doi.org/10.1007/s12350-009-9187-5

[16] Snapper HJ, Shea NL, Konstam MA. Combined analysis of resting regional wall thickening and stress perfusion with electrocardiographic-gated technetium 99m-labeled sestamibi single-photon emission computed tomography: Prediction of stress defect reversibility. J Nucl Cardiol. 1997; 4: 3-10. http://dx.doi.org/10.1016/S1071-3581(97)90043-X

[17] Heo J, Kegel J, Iskandrian AS et al. Comparison of same- using Technetium-99-m-sestamibi myocardial imagning. J Nucl Med. 1992; 33: 186-91. PMid:1531069

[18] Cheetham AM, Naylor V, McGhie J et al. Is stress only imaging practical when a 1-day stress rest 99mTc tetrofosmin protocol is use? Nucl Med Commun. 2006; 27: 113-7. PMid:16404223 http://dx.doi.org/10.1097/01.mnm.0000191854.11704.77

[19] Tägil K, Lomsky M, Marving J. Use of neural networks to improve quality control of interpretations in myocardial perfusion imaging. Int J Cardiovasc Imaging. 2008; 24: 841-848. PMid:18587683 http://dx.doi.org/10.1007/s10554-008-9329-x

[20] Hendel RC. Attenuation correction: eternal dilemma or real improvement? Q J Nucl Med Mol Imaging. 2005 ; 49 : 30-42. PMid:15724134 